\title{
Relative timing jitter of Raman soliotns and its effect on nonlinear wavelength conversion
}

\author{
Gengji Zhou, ${ }^{1}$ Ming Xin, ${ }^{1}$ Franz X. Kärtner,,${ }^{1,2,3,4}$ and Guoqing Chang ${ }^{1,3,4, *}$ \\ ${ }^{1}$ Center for Free-Electron Laser Science, DESY, Notkestraße 85, 22607 Hamburg, Germany \\ ${ }^{2}$ Physics Department, University of Hamburg, Luruper Chaussee 149, 22761 Hamburg, Germany \\ ${ }^{3}$ The Hamburg Centre for Ultrafast Imaging, Luruper Chaussee 149, 22761 Hamburg, Germany \\ ${ }^{4}$ Department of Electrical Engineering and Computer Science and Research Laboratory of Electronics, \\ Massachusetts Institute of Technology, 77 Massachusetts Avenue, Cambridge, MA 02139, USA \\ "guoqing.chang@desy.de
}

\begin{abstract}
We demonstrate that shorter fiber length and the excitation pulse with shorter duration and higher energy can reduce a Raman soliton's relative timing jitter and leads to reduced noise in nonlinear wavelength conversion.

(C)2015 Optical Society of America

OCIS codes: (190.4370) Nonlinear optics, fibers; (320.7110) Femtosecond phenomena.
\end{abstract}

When a femtosecond pulse propagates inside an optical fiber with anomalous dispersion, interplay between intrapulse stimulated Raman scattering and soliton formation results in Raman soliton self-frequency shift; that is, the Raman soliton's center frequency red shifts continuously during propagation and maintains its transform-limited hyperbolic secant profile. Capable of providing wavelength tunable femtosecond pulses at the desired wavelengths that cannot be directly obtained from mode-locked ultrafast lasers, Raman soliton sources have found many important applications. As a particular example of nonlinear wavelength conversion, difference-frequency generation (DFG) between a Raman soliton and the pumping pulse that excites the Raman solliotn constitutes a powerful method to generate mid-IR femtosecond pulses [1]. Since the Raman soliton and its excitation pulse share the same repetition rate and the carrier-envelope phase (CEP) offset, the resulting DFG source-if the excitation pulse's repetition rate is stabilized - becomes a mid-IR frequency comb with its CEP offset automatically set at zero. Raman soliton's center wavelength can be tuned by varying the excitation pulse's energy, and therefore the relative intensity noise (RIN) of the excitation pulse causes the Raman soliton's center-wavelength fluctuation, which is then converted to timing jitter by fiber dispersion. In the subsequent DFG, this relative timing jitter between the excitation pulse and the Raman soliton will be transferred to the derived mid-IR comb source manifesting as broadened comb lines and increased RIN. In this paper, we carried out a detailed experimental study on optimizing Raman soliton's relative timing jitter and the resulting effect on nonlinear wavelength conversion.
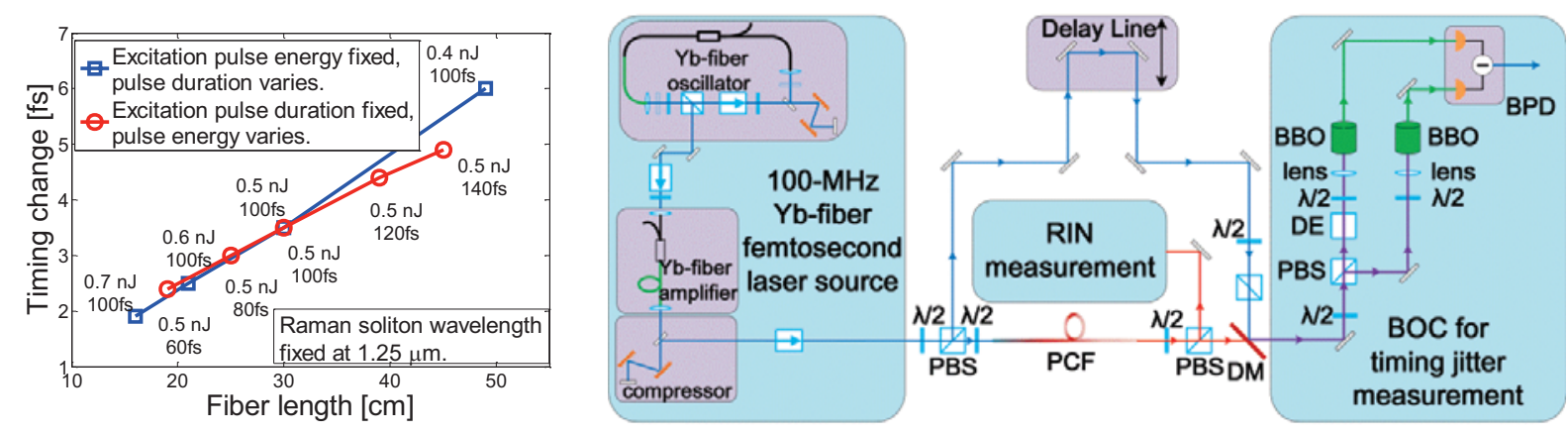

Fig 1. (left) Simulation results for Raman soliton's timing change due to $0.2 \%$ change of the excitation pulse energy. A $1.03 \mu \mathrm{m}$ excitation pulse propagates in a photonic crystal fiber with zero-dispersion wavelength at $945 \mathrm{~nm}$ and the resulting Raman soliton's center wavelength is fixed at $1.25 \mu \mathrm{m}$. (right) Schematic setup to characterize relative timing jitter of a Raman soliton source. BOC: balanced optical correlator, PBS: polarization beam splitter, PCF: photonic crystal fiber, DM: dichroic mirror, DE: delay element, BPD: balanced photodetector.

Raman soliton centered at a desired wavelength can be achieved by different combination of fiber length and the excitation pulse's duration and energy. Figure 1(left) shows - by solving the generalized nonlinear Schrödinger equation-nine possible parameter-combinations that result in a Raman soliton at the same wavelength of $1.25 \mu \mathrm{m}$ using a photonic crystal fiber (PCF) with its zero-dispersion wavelength at $945 \mathrm{~nm}$. The excitation pulse's center wavelength is fixed at $1.03 \mu \mathrm{m}$. We vary the excitation pulse energy by $0.2 \%$ and record the corresponding timing change of the Raman soliton. As Fig. 1(left) illustrates, increasing excitation pulse energy (with duration fixed at 
$100 \mathrm{fs}$, blue curve) or reducing the pulse's duration (with energy fixed at $0.5 \mathrm{~nJ}$, red curve) both lead to (1) a shorter required PCF length to generate a Raman soliton centered at $1.25 \mu \mathrm{m}$, and (2) a decreased timing change. It suggests that a combination of shorter excitation pulse, higher excitation pulse energy, and shorter fiber length can reduce the relative timing jitter between a Raman soliton and its excitation pulse. Guided by numerical simulations, we use the experimental setup shown in Fig. 1(right) to study the relative timing jitter of Raman solitons shifted to $1225 \mathrm{~nm}$ and $1275 \mathrm{~nm}$ using two PCF (zero-dispersion wavelength at $945 \mathrm{~nm}$ ) lengths $-38 \mathrm{~cm}$ and $205 \mathrm{~cm}$. The home-built $\mathrm{Yb}-$ fiber laser system providing 3-nJ, 110-fs pulses includes a 100-MHz Yb-fiber oscillator centered at $1035 \mathrm{~nm}$, an Ybfiber amplifier, and a pulse compressor. We constructed a balanced optical correlator (BOC) [2] to measure the relative timing jitter between the excitation pulse and the Raman soliton.
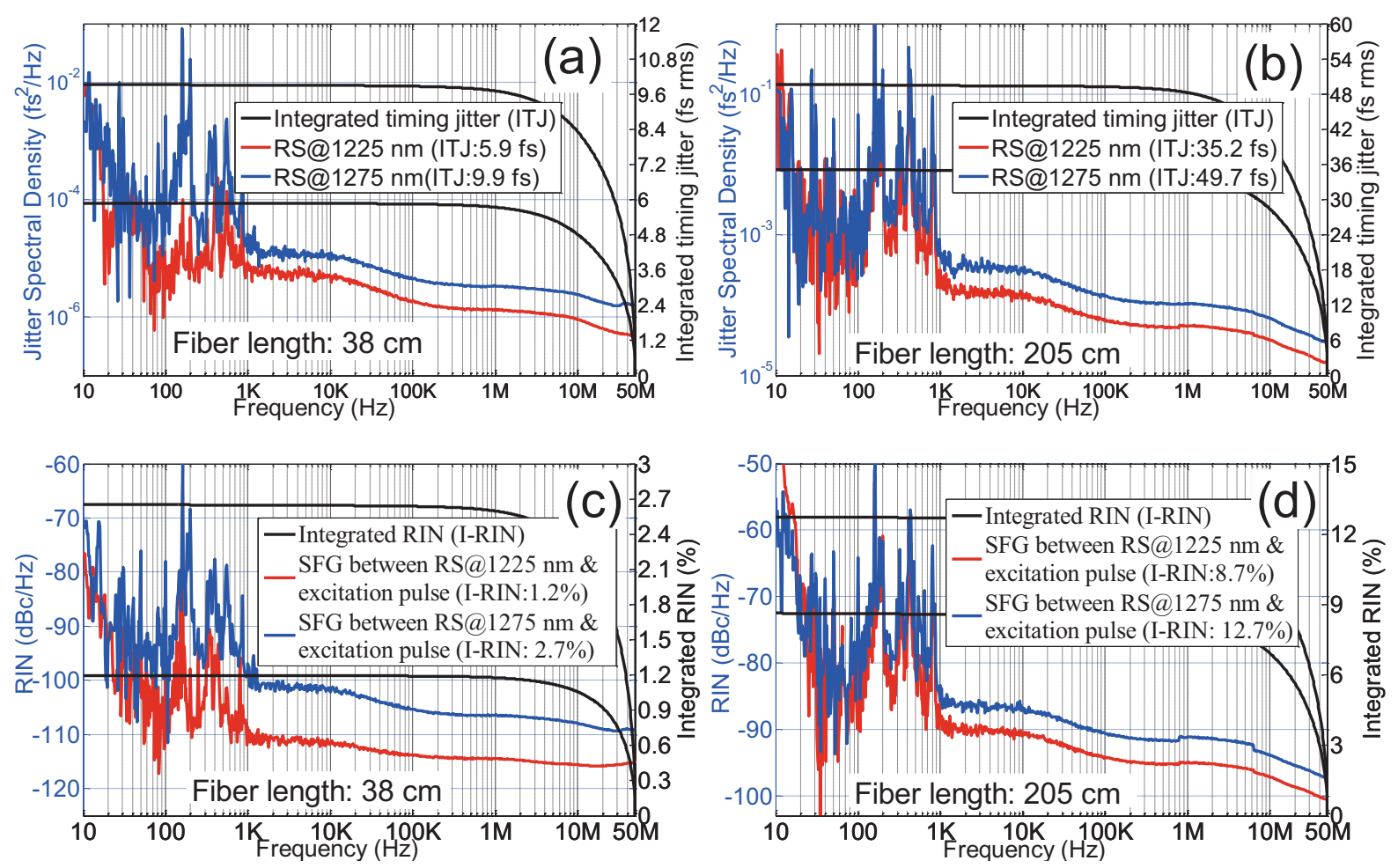

Fig 2. (a)(b) relative timing jitter of Raman solitons (RS) centered at $1225 \mathrm{~nm}$ (red curve) and $1275 \mathrm{~nm}$ (blue curve) using 38-cm PCF (a) and 205-cm PCF (b). Black curves are the integrated timing jitter (ITJ). (c)(d) RIN measurement of SFG pulse generated by mixing the excitation pulse with the 1225-nm Raman soliton (red curve) or with the 1275-nm Raman soliton (blue curve). (c) results from 38-cm PCF and (d) results from 205-cm PCF. Black curves represent the integrated RIN.

Figure 2(a) and 2(b) plot the relative timing jitter of two Raman solitons peaking at different wavelengths (1225 $\mathrm{nm}$ versus $1275 \mathrm{~nm}$ ) generated by 38-cm PCF (Fig. 2(a)) and by 205-cm PCF (Fig. 2(b)). As expected from simulation, using shorter fiber length (i.e., $38 \mathrm{~cm}$ ) with larger excitation pulse energy reduces timing jitter. More specific, the integrated timing jitter from $50 \mathrm{MHz}$ to $10 \mathrm{~Hz}$ is 35.2 (49.7) fs for the 1225- (1275-) nm Raman soliton generated from 205-cm PCF; this value drops to 5.9 (9.9) fs when 38-cm PCF is employed. To study the effect of the relative timing jitter on nonlinear wavelength conversion, we measured the RIN of the sum-frequency generation (SFG) between Raman solitons and the excitation pulse. The SFG pulse exhibits an order of magnitude higher RIN (Fig. 2(c) and 2(d)) than the excitation pulse and the resulting Raman soliton, both of which have an integrated RIN around $\sim 0.2 \%$. Apparently it is the relative timing jitter that generates such a substantial increase of the RIN for these SFG pulses. As expected from Fig. 2(c)(d), a reduction of relative timing jitter using the 38 -cm PCF reduces the SFG pulses' integrated RIN by a factor of $\sim 5$ compared with the results from the 205 -cm PCF. Ongoing work is to reduce this relative timing jitter to the attosecond level by further optimization and achieve a low-noise femtosecond source derived from nonlinear mixing between a Raman soliton and the excitation pulse.

\section{References}

1. N. Coluccelli et al., Opt. Lett. 39, 1661 (2014); A. Gambetta et al., Opt. Lett. 38, 1155 (2013); T. Steinle et al., Opt. Express 22, 9567 (2014); D. G. Winters et al., Opt. Lett. 35, 2179 (2010); T. W. Neely et al., Opt. Lett. 36, 4020 (2011).

2. J. Kim, et al., Opt. Lett. 32, 3519-3521 (2007). 